\title{
Enhanced Coronary Vasa Vasorum Neovascularization in Experimental Hypercholesterolemia
}

\author{
Hyuck Moon Kwon, Giuseppe Sangiorgi, Erik L. Ritman, ${ }^{\star}$ Charles McKenna, David R. Holmes, Jr., Robert S. Schwartz, \\ and Amir Lerman \\ Division of Internal Medicine and Cardiovascular Diseases, and the *Department of Physiology and Biophysics, Mayo Clinic, Rochester, \\ Minnesota 55905
}

\begin{abstract}
Coronary arteries contain a network of vasa vasorum in the adventitia. The three-dimensional anatomy of the vasa vasorum in early coronary atherosclerosis is unknown. This study was designed to visualize and quantitate the threedimensional spatial pattern of vasa vasorum in normal and experimental hypercholesterolemic porcine coronary arteries, using a novel computed tomography technique. Animals were killed after being fed either a high cholesterol diet $(n=4)$ or a control diet $(n=4)$ for $12 \mathrm{wk}$. The proximal left anterior descending coronary artery was removed from the heart, scanned, and reconstructed, and quantitation of vasa vasorum density was performed. Two different types of vasa vasorum were defined: first-order vasa vasorum ran longitudinally parallel to the vessel and second-order originated from first-order vasa circumferentially around the vessel wall. Compared with controls in hypercholesterolemic coronary arteries, there was a significant increase in the area of the vessel wall $\left(3.86 \pm 0.22\right.$ vs. $8.07 \pm 0.45 \mathrm{~mm}^{2}$, respectively, $P<0.01$ ) and in the density of vasa vasorum $\left(1.84 \pm 0.05 / \mathrm{mm}^{2}\right.$ vs. $4.73 \pm 0.24 / \mathrm{mm}^{2}$; respectively, $\left.P=0.0001\right)$. This occurred especially by an increase of second-order vasa vasorum and disorientation of normal vasa vasorum spatial pattern. This study suggests that adventitial neovascularization of vasa vasorum occurs in experimental hypercholesterolemic coronary arteries and may be a part of the early atherosclerotic remodeling process. (J. Clin. Invest. 1998. 101:1551-1556.) Key words: atherosclerosis • circulation • adventitia • imaging • microscopic computed tomography
\end{abstract}

\section{Introduction}

In human and animal coronary arteries, a fine network of microvasculature termed vasa vasorum is normally present in the adventitial layer, presumably to provide oxygen and nutrients to the outer layers of the arterial wall (1). Experimental and human pathologic studies have shown an increased number of adventitial vasa vasorum in advanced coronary atherosclerosis (2-5). Recently, a correlation between the extent of vasa vasorum neovascularization and severity of atherosclerotic dis-

Address correspondence to Amir Lerman, M.D., Division of Cardiovascular Diseases, Mayo Clinic, 200 First St. SW, Rochester, MN 55905. Phone: 507-255-4152; FAX: 507-255-2550; E-mail: Lerman.Amir@ mayo.edu

Received for publication 21 August 1997 and accepted in revised form 4 February 1998.

The Journal of Clinical Investigation

Volume 101, Number 8, April 1998, 1551-1556

http://www.jci.org ease has been demonstrated in human coronary arteries (2). Moreover, in animal studies, injury of the adventitial layer has been shown to stimulate proliferation of these vessels (6-8). Thus, these observations indicate that adventitial vasa vasorum may play a role in the development and the progression of coronary atherosclerosis. However, whether proliferation of the vasa vasorum precedes or follows plaque formation is still not known.

There is a lack of information regarding the spatial three dimensional pattern of the vasa vasorum in the coronary artery. This is due to the technical difficulties in visualizing the course of this fine vascular network with histologic methods. Microscopic computed tomography (micro-CT) ${ }^{1}$ is a novel technique that allows three-dimensional views of the entire microvascular structure (9-12). By this technique it is possible to reconstruct the spatial distribution of vessels within an organ (9-12).

This study was designed to test the hypothesis that early coronary atherosclerosis before the development of vascular lesion is associated with changes in the spatial distribution of adventitial vasa vasorum. To accomplish this aim we visualized and quantitated the coronary adventitial vasa vasorum during experimental hypercholesterolemia, which resembles the structural and functional changes present in the early phase of coronary atherosclerotic development (13-15), using a threedimensional micro-CT technique.

\section{Methods}

Animals and arterial specimens. The following study was performed after approval of the Mayo Clinic Institutional Animal Care and Use Committee. The experiment was conducted using eight female juvenile domestic cross-bred pigs weighing $25-35 \mathrm{~kg}$. Four pigs were placed on a normal laboratory chow diet (group 1). Another four animals were fed a high-cholesterol diet of $2 \%$ cholesterol and $15 \%$ lard by weight (TD 93296; Harlan Teklad) for 12 wk (group 2). Plasma total cholesterol and lipoprotein levels were determined by an enzymatic method previously described (16), using a commercial reagent (Roche Laboratories, Division of Hoffman-La Roche Inc., Nutley, $\mathrm{NJ})$. After plasma cholesterol level measurements, the animals were killed using an intravenous commercial euthanasia solution by ear vein (10 ml Sleepaway; Fort Dodge Laboratories, Fort Dodge, IA). Then, the heart was removed and its size and weight measured.

Coronary artery perfusion and glycerin dehydration. After removal of the heart, glass cannulae were tied at the coronary orifices and injected with $500 \mathrm{ml}$ of heparinized saline $(0.9 \%$ sodium chloride with $5,000 \mathrm{U}$ of heparin) at a pressure of $70 \mathrm{mmHg}$ in order to clear the coronary network of the remaining blood. A low-viscosity, radiopaque liquid polymer compound (MV-122; Canton Biomedical Products, Boulder, $\mathrm{CO}$ ) was then injected through the cannulae and the heart was then immersed in $10 \%$ buffered formalin and placed under

1. Abbreviation used in this paper: micro-CT, microscopic computed tomography. 
refrigeration at $4^{\circ} \mathrm{C}$ overnight to allow polymerization of compound. On the following day, the proximal part of the left anterior descending coronary artery ( $\sim 2 \mathrm{~cm}$ long) was removed by careful dissection and placed in a $95 \%$ alcohol solution for $48 \mathrm{~h}$. At successive $24-\mathrm{h}$ intervals, the glycerin concentration was raised from $30,50,75 \%$, and finally pure glycerin in order to completely dehydrate the coronary segments. Then, the specimen was rinsed with acetone, left in the open air for $24 \mathrm{~h}$, and embedded in a paraffin mold for three-dimensional micro-CT imaging.

Microscopic three-dimensional CT imaging and reconstruction. Specimens were scanned by a micro-CT system consisting of a spectroscopy $x$-ray tube, a fluorescent crystal plate, a microscope objective, and a charge-coupled device camera (9-11). The charge in each pixel was digitized and stored as an array in a computer as $500-1,000$ projections of the specimen in $360^{\circ}$ rotations. Three-dimensional images were reconstructed using a modified Feldhaup cone beam filtered back projection algorithm and the resulting three-dimensional images displayed using Mayo Analyze software (version 7.5; Biomedical Imaging Resource, Mayo Foundation). Volume rendering provided a variety of display representations of three-dimensional image data sets. Volume-rendered transmission displays, maximum intensity projection, and voxel gradient shading were displayed at various angles and threshold values of voxels. Average voxel size was 21-28 $\mu \mathrm{m}$, and images of up to 800 slices were rendered for each arterial specimen (each with a matrix of 10-20 $\mu \mathrm{m}$ cubic voxels $\times 16$ bits of gray scale).

From each specimen 7-10 cross-sectional images were analyzed every $2 \mathrm{~mm}$, at the pixel size of $21 \mu \mathrm{m}$ (normal sections, $n=39$; hypercholesterolemic sections, $n=36$ ). Segment branching points were intentionally excluded from the analysis since these sites could be a source of variation in vasa vasorum density.

The boundary of the coronary arterial wall containing vascular structures was defined using a radius of twice the distance from the arterial lumen to the outer adventitia (17). The blood vessels within this boundary were determined as vasa vasorum. The area of vasa vasorum within the vessel wall area was differentiated from nonvascular structures by setting lower threshold values for an intensity range of interest that yielded the best identification of vasa vasorum regions as judged independently by two operators (H.M. Kwon and G. Sangiorgi). Then, the number of vasa vasorum was manually counted and divided by the total area of vessel wall to obtain the density of vasa vasorum $\left(\mathrm{No} . / \mathrm{mm}^{2}\right)$.

Two anatomically different types of vasa vasorum were visually defined (18). First-order vasa vasorum originated from the main coronary lumen and ran longitudinally parallel to the coronary artery. Second-order vasa vasorum originated from first-order vasa vasorum or branches and ran circumferentially around the lumen, as arch arterioles. The number and diameter of those vessels were counted and measured separately. In addition, to confirm the integrity of the vasa vasorum, first- and second-order vasa were followed from their origin for the entire length along the main coronary artery lumen.

After visually determining the three-dimensional pattern of vasa vasorum, the following morphometric variables were calculated: $(a)$ vessel wall area, defined as a radius of twice the distance from the arterial lumen to the outer adventitia; $(b)$ density of vasa vasorum per square millimeter of vessel wall area; $(c)$ ratio of first- to second-order vasa vasorum; and $(d)$ mean diameter of first- and second-order vasa vasorum. Finally, a segment-by-segment analysis was performed to assess variation in the vasa vasorum number and in the area of vessel wall for a given segment along the entire length of the coronary artery segments.

Histology. After micro-CT reconstruction and analysis, coronary specimens were immersed for $4 \mathrm{~h}$ in $40^{\circ} \mathrm{C}$ hot water to gently melt the wax embedding, removed from the plastic mold, cut every $2 \mathrm{~mm}$, and stained with hematoxylin and eosin and elastic van Gieson. The number of vasa in the vessel wall area was determined on histologic crosssections for correlation with corresponding micro-CT cross-sectional images.
Statistical analysis. All data in the text and figures are presented as mean \pm SEM. Data were analyzed using unpaired $t$ test and oneway ANOVA to establish differences among groups. Correlations between the continuous variables was analyzed using a linear regression model. A value of $P<0.05$ was considered significant in all analyses.

\section{Results}

No statistically significant differences were observed in the size and weight of the hearts between normal and hypercholesterolemic animals. There was a significant increase in plasma cholesterol (341 \pm 75 vs. $106 \pm 11 \mathrm{mg} / \mathrm{dl}, P<0.01)$ and low-density lipoprotein levels $(263 \pm 72$ vs. $34 \pm 20 \mathrm{mg} / \mathrm{dl}, P<0.01)$ in highcholesterol fed animals compared with controls.

Vasa vasorum in normal coronary arteries. Morphometric data for normal and injured coronary segments are summarized in Table I. Voxel gradient shading images showed that normal vasa vasorum originated from coronary artery branch points at regular intervals and ran longitudinally along the vessel wall (first-order vasa vasorum). These vessels then separated to form circumferential arches around the main coronary lumen (second-order vasa vasorum, Fig. 1). The density of vasa vasorum in normal coronary arteries was $1.84 \pm 0.05 / \mathrm{mm}^{2}$ and the density ratio of first- to second-order vasa was 3:2 (Table I). Normal first- and second-order vasa vasorum differed in the mean diameter value $(160.92 \pm 5.10$ vs. $67.99 \pm 2.72 \mu \mathrm{m}, P=$ 0.0001, respectively, Table I).

Vasa vasorum in experimental hypercholesterolemic coronary arteries. Porcine coronary arteries obtained from experimental hypercholesterolemia showed a dense plexus of microvessels in the adventitia, resulting in a disorientation of the normal vasa vasorum pattern. Such structures were not found in normal coronary arteries (Fig. 2).

Experimental hypercholesterolemic coronary arteries showed increased vessel wall area compared with controls $(8.07 \pm 0.45$ vs. $3.86 \pm 0.22 \mathrm{~mm}^{2}$; respectively, $P=0.0001$, Table I and Fig. 3). Morphologic quantitation documented significantly higher spatial vasa vasorum density in hypercholesterolemic coronary arteries compared with normal coronary arteries $(4.73 \pm 0.24$ / $\mathrm{mm}^{2}$ vs. $1.84 \pm 0.05 / \mathrm{mm}^{2}, P=0.0001$, Fig. 4$)$. The diameter of first-order vasa was also decreased $(107.7 \pm 2.49$ vs. $160.92 \pm$ $5.10 \mu \mathrm{m}, P=0.0001$, Table I). New vessel formation occurred especially by an increase of second-order vasa vasorum, with a shift from first- to second-order vasa (normal 3:2 vs. hypercholesterolemic 1:2, Table I). A significant correlation was present

Table I. Results of Quantitative Computerized Digital Analysis of Micro-CT Images by Normal and Experimental Hypercholesterolemic Coronary Arteries

\begin{tabular}{lcc}
\hline & Normal & Hypercholesterolemia \\
\hline & $n=39$ & $n=36$ \\
Vessel wall area $\left(\mathrm{mm}^{2}\right)$ & $3.86 \pm 0.22$ & $8.07 \pm 0.45^{*}$ \\
Density of vasa vasorum $\left(\mathrm{No} . / \mathrm{mm}^{2}\right)$ & $1.84 \pm 0.05$ & $4.73 \pm 0.24^{*}$ \\
Ratio of $1^{\text {st }} / 2^{\text {nd }}$ order vasa density & $3: 2$ & $1: 2^{*}$ \\
Mean diameter of $1^{\text {st }}$ order vasa $(\mu \mathrm{m})$ & $160.92 \pm 5.10$ & $107.75 \pm 2.49^{*}$ \\
Mean diameter of $2^{\text {nd }}$ order vasa $(\mu \mathrm{m})$ & $67.99 \pm 2.72$ & $73.54 \pm 1.34$
\end{tabular}

$* P<0.01$. 


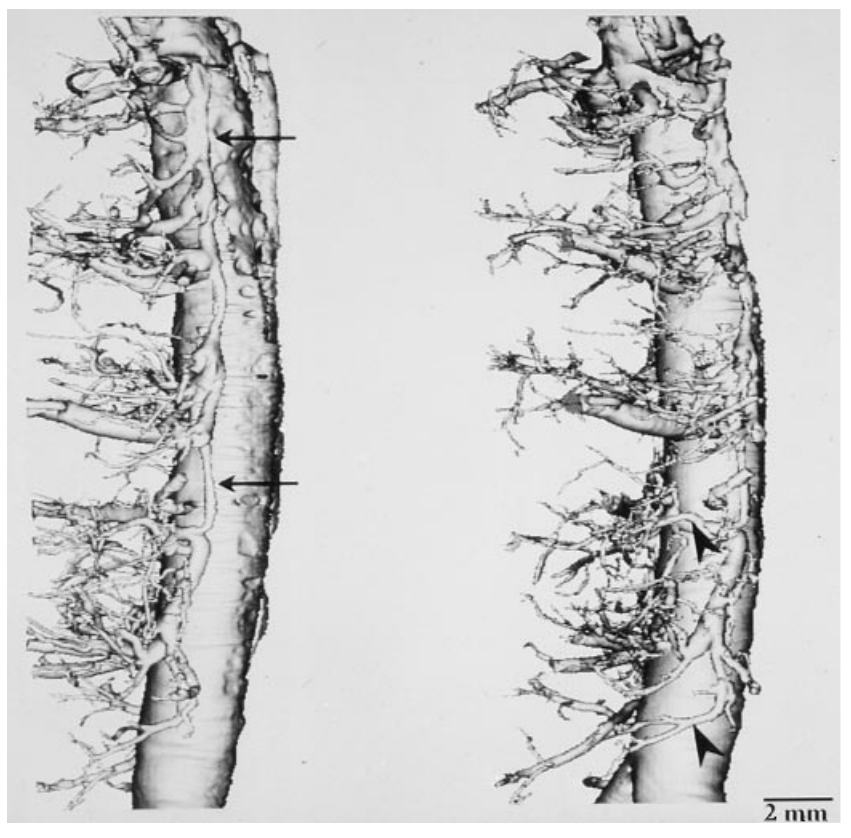

Figure 1. Micrographs showing voxel gradient shading of normal coronary artery at different angles. Two anatomically different vasa types can be seen. First-order vasa vasorum (arrows) originate from branches of coronary artery, and run longitudinally to the main lumen. Second-order vasa vasorum (arrowheads) originate from firstorder vasa, and run circumferentially around the lumen, forming arch arterioles. Voxel size, $28 \mu \mathrm{m}$.

between the number of vasa vasorum and wall area in hypercholesterolemic coronary arteries $(r=0.71, P<0.0004)$. At the segmental analysis, as the arterial wall became thicker, the number of vasa vasorum also increased. In areas where no increase in wall thickness was present, no proliferation of vasa vasorum was observed.
At the histologic examination, arteriolar vasa vasorum appeared as black areas filled with by the radiopaque polymer (Fig. 3). The number and distribution of arteriolar vasa vasorum with cross-sectional micro-CT images was similar between micro-CT and histological sections.

\section{Discussion}

This study was designed to investigate the effects of experimental hypercholesterolemia on adventitial vasa vasorum in porcine coronary arteries. We outlined, for the first time, the three-dimensional anatomy of vasa vasorum in normal and hypercholesterolemic porcine coronary arteries using a powerful novel imaging technique. The results suggest the following conclusions: $(a)$ in normal pig coronary arteries two principal anatomic distinguished vasa vasorum types were present; $(b)$ experimental hypercholesterolemia is characterized by a significant increase in the coronary vessel wall area and in the density of adventitial vasa vasorum; and (c) there was a disorientation of the normal three-dimensional spatial pattern characterized by a shift from first- to second-order vasa. This study suggests that in experimental hypercholesterolemia there is increased vasa vasorum neovascularization in the adventitia layer of porcine coronary arteries before the development of vascular lesions.

Several investigators have shown that the tunica adventitia of coronary arteries contains a fine network of vessels $(1,3,6)$, but no methods have been available to quantitate vasa vasorum using a detailed morphological approach. The limited spatial resolution of two-dimensional techniques precludes in fact a more sophisticated analysis of the course of these vessels. Micro-CT is a novel and powerful technique that permits the assessment of the three-dimensional pattern of microvascular structure and provides a useful means for the study of the spatial distribution of vessels within an organ (9-12). In this study, the utilization of this technique allowed us to identify the origin and follow the course of first- and second-order vasa vasorum in coronary arteries of normal and hypercholesterolemic pigs. Normal first-order vasa vasorum originated at reg-

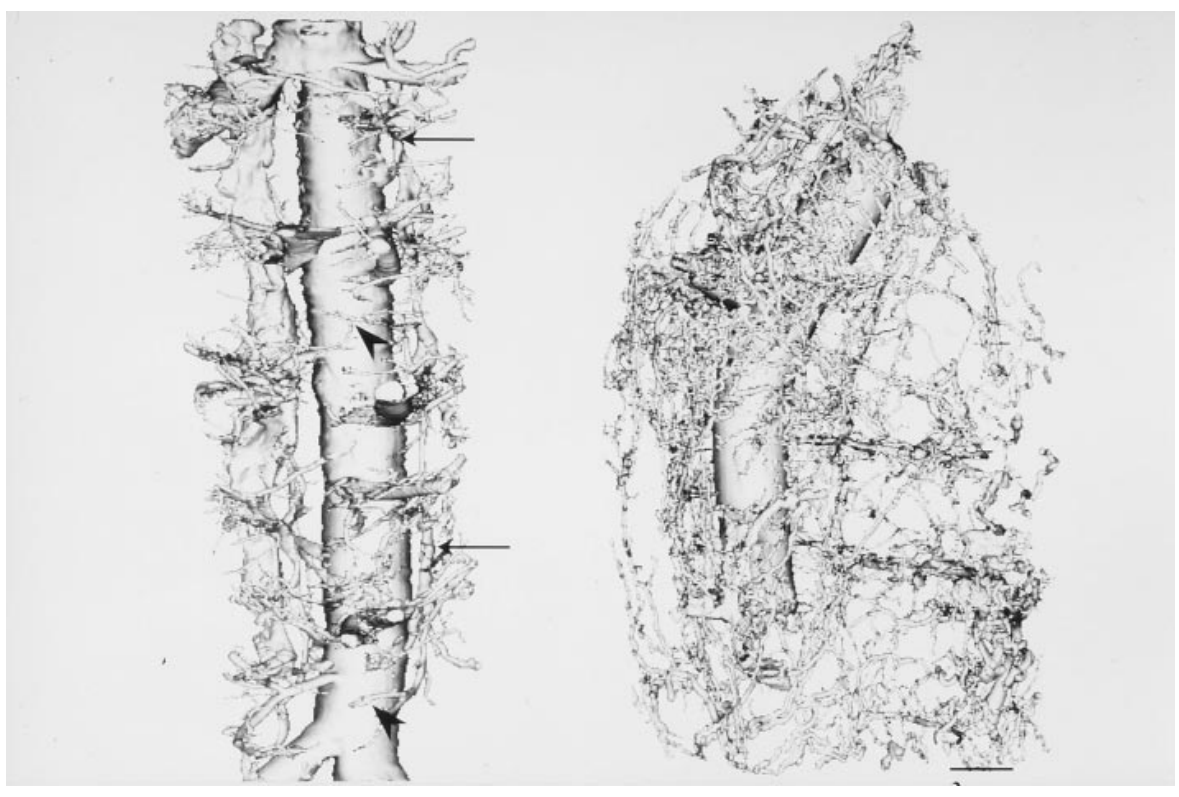

Figure 2. Micrographs showing the spatial distribution of adventitial vasa vasorum in voxel gradient shading from normal (left) and experimental hypercholesterolemic coronary artery (right). Coronary artery obtained from experimental hypercholesterolemic animals showed a dense plexus of microvessels in the adventitia. In addition there was disorientation of the normal three-dimensional spatial pattern. Firstorder vasa vasorum are indicated by arrows. Second-order vasa vasorum are indicated by arrowheads. Voxel size, $28 \mu \mathrm{m}$. Bar size, $2 \mathrm{~mm}$. 

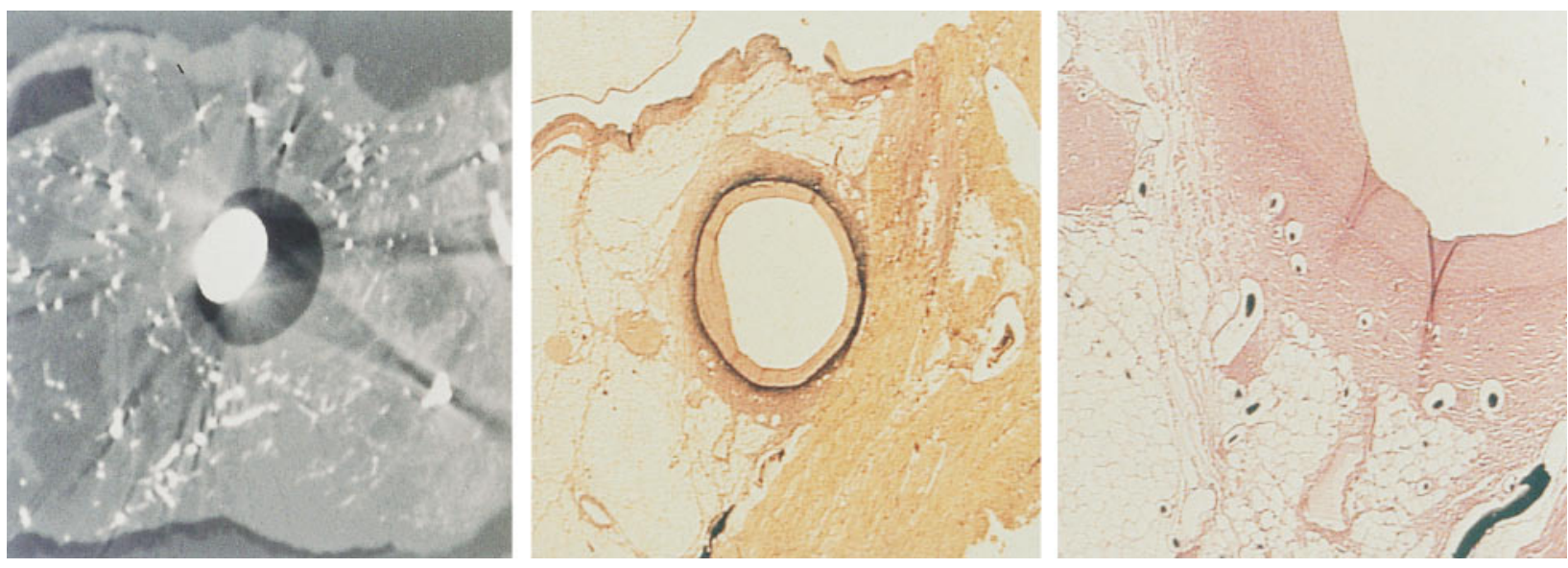

Figure 3. Micrographs showing micro-CT cross sectional images (left, voxel size $21 \mu \mathrm{m}$ ) compared with histologic cross-sectional images (elastic van Gieson staining, $\times 4$, middle; hematoxylin and eosin staining, $\times 10$, right $)$. A good correspondence is present between micro-CT and histologic cross-sectional images. Arteriolar vasa vasorum are filled with polymer (black) in the histologic sections.

ular intervals from the coronary artery along the vessel wall and then separated to give origin to second-order vasa which run circumferentially around the arterial lumen. Conversely, in coronary arteries from hypercholesterolemic animals, a newly formed, dense plexus of finely anastomosing microvessels, principally second-order vasa vasorum, was present in the adventitia. The three-dimensional pathway of these vessels was totally disorganized. The potential pathogenetic role of these neovessels in the development and maintenance of coronary atherosclerosis is not known. It is possible that this abundant vasa vasorum plexus may be of profound functional significance, in that it may create a plane where low blood pressure is present, thus, promoting an increased transmural flux of plasma nutrients $(19,20)$.

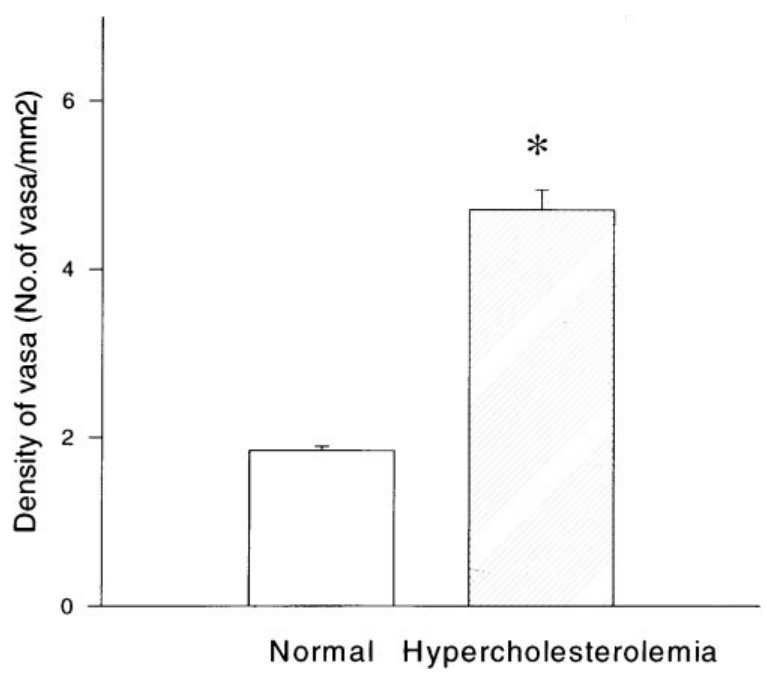

Figure 4. Bar graphs showing the mean density of adventitial vasa vasorum (No. vasa/ $\mathrm{mm}^{2}$ ) in normal and hypercholesterolemic coronary arteries. Morphologic quantitation documented significantly higher vasa vasorum density in experimental hypercholesterolemic coronary arteries compared with normal controls. $* P=0.0001$.
A direct relationship between severity of atherosclerosis and the degree of adventitial vasa vasorum is well established $(2,4)$ and recent studies suggest that the primary stimulus linking these two processes is an hypoxic state either related to an increase in arterial wall thickness or to adventitial vasa vasorum damage $(6,21)$. Specifically, several investigators have observed an increased density of vasa vasorum in regions of atherosclerotic plaques $(2,3)$. In a recently described model of atherogenesis, compression of the adventitia layer by placement of a soft silastic collar around rabbit carotid arteries induced focal intimal hyperplasia (7). Furthermore, in different animal models either occlusion or removal of adventitial vasa vasorum has been shown to cause intimal hyperplasia. This finding is probably related to hypoxia of the arterial wall $(6,22$, 23) which regresses with generation of a new adventitial layer (24). A reduction of coronary artery vasa vasorum during regression of atherosclerosis has been demonstrated (4). These observations may also be related to a decrease in thickness of vessel wall and restoration of adequate nutrient diffusion from the adventitia and the lumen of the coronary artery.

In our study, experimental hypercholesterolemia induced a significant increase in coronary vessel wall area. Even though this morphological change does not represent classic atherosclerotic lesions per se, it resembles the structural changes present in the early phase of coronary atherosclerotic development (13-15). In addition, the number of newly formed vasa increased proportionally with the increase in coronary wall area. As shown by the segmental analysis, as the coronary wall area increased, so did the number of vasa vasorum.

It is known that the arterial wall can receive nutrients by diffusion outwards from the main lumen and inwards by adventitial vasa vasorum $(1,25-27)$. If the vessel wall thickness exceeds a "critical depth" $(1,25)$ then the oxygen supply to the media is provided by newly formed vasa vasorum within this layer. The increased thickness of the arterial wall due to an hypercholesterolemic state may produce a relatively hypoxic state in the media layer which in turn triggers vasa vasorum formation in high-cholesterol fed animals. This hypothesis is supported by the fact that in regions where no increase in vessel wall area was observed, there was also a lack of vasa va- 
sorum formation. If this hypothesis is correct, then reversing wall hypoxia may cause arterial wall thickness to regress. On the other hand, interfering with the vasa vasorum blood supply by an antiangiogenic therapy might also induce a reduction in wall thickness (28), as demonstrated by regression studies on atherosclerotic plaques $(4,24)$.

The mechanisms underlying early proliferation especially of the second-order vasa vasorum during atherosclerotic lesion development have not been understood yet. Experimental hypercholesterolemia may induce upregulation of specific cellsurface receptors (29-32) in the second-order vasa which then predisposes them to proliferate in response to growth factors $(17,32,33)$. Specifically, it has been demonstrated that the low-density lipoprotein receptor-related protein $/ \alpha_{2}$-macroglobulin receptor, which is present normally in endothelial cells of vasa vasorum and smooth muscle cells of the media (34), may play an important local scavenger role for certain cytokines/ growth factors $(35,36)$. Recently, Jamieson and co-authors have demonstrated a correlation between apo(a) deposition within endothelial cells of vasa vasorum and the stage of atherosclerotic plaque in human cerebral vessels (37), suggesting a specific link between apo(a) receptor and the pathogenesis of atherosclerosis. Increased angiogenic factor expression in the adventitia has been demonstrated by several investigators $(4,24,26,38-40)$. In addition, certain angiogenic factors are also vascular smooth muscle cell and endothelial mitogens (17, 39, 41, 42). Further experimental studies are needed to better understand the relationship between growth factor upregulation and second-order vasa vasorum formation in coronary atherosclerosis.

Conclusions. This study suggests that adventitial neovascularization of vasa vasorum occurs in experimental hypercholesterolemic coronary arteries before the development of vascular lesion. This occurred especially by an increase of second-order vasa and a disorientation of the normal vasa vasorum pattern, which may be a part of the early atherosclerotic remodeling process.

\section{Acknowledgments}

This study was supported by the National Institutes of Health (HL03180-01), the Miami Heart Research Institute, The Bruce and Ruth Rappaport Vascular Biology Program, and the Mayo Foundation.

\section{References}

1. Wolinsky, H., and S. Glagov. 1967. Nature and species differences in the medial distribution of aortic vasa vasorum in mammals. Circ. Res. 20:409-421.

2. Kumamoto, M., Y. Nakashima, and K. Sueishi. 1995. Intimal neovascularization in human atherosclerosis. Its origin and pathophysiological significance. Hum. Pathol. 26:450-456.

3. Barger, A.C., R. Beeuwkes, L.L. Lainey, and K.J. Silverman. 1984. Hypothesis: vasa vasorum and neovascularization of human coronary arteries. $N$. Engl. J. Med. 310:175-177.

4. Williams, J.K., M.L. Armstrong, and D.D. Heistad. 1988. Vasa vasorum in atherosclerotic coronary arteries. Responses to vasoactive stimuli and regression of atherosclerosis. Circ. Res. 62:515-523.

5. Zamir, M., and M.D. Silver. 1985. Vasculature in the walls of human coronary arteries. Arch. Pathol. Lab. Med. 109:659-662.

6. Barker, S.G.E., A. Talbert, S. Cottam, P.A. Baskerville, and J.F. Martin. 1993. Arterial intimal hyperplasia after occlusion of the adventitial vasa vasorum in the pig. Arterioscler. Thromb. 13:70-77.

7. Booth, R.F.G., J.F. Martin, A.C. Honey, D.G. Hassall, J.E. Beesley, and S. Moncada. 1989. Rapid development of atherosclerotic lesions in rabbit carotid artery induced by perivascular manipulation. Atherosclerosis. 76:257-268

8. Martin, J.F., R.F. Booth, and S. Moncada. 1991. Arterial wall hypoxia fol- lowing thrombosis of the vasa vasorum is an initial lesion in atherosclerosis. Eur. J. Clin. Invest. 21:355-359.

9. Flannery, B.P., H.W. Dickman, W.G. Roberge, and K.L. D'Amico. 1987. Three-dimensional x-ray micro tomography. Science. 237:1439-1444.

10. Avula, R.T.V., J.H. Dunsmuir, P.E. Beighley, P.J. Thomas, A. Faridani, and E.L. Ritman. 1994. A micro tomographic technique enhanced by novel image reconstruction algorithm: application to rat coronary vessels. FASEB (Fed. Am. Soc. Exp. Biol.) J. 8:A854. (Abstr.)

11. Ritman, E.L., J.H. Dunsmuir, A. Faridani, D.V. Finch, K.T. Smith, and P.J. Thomas. 1997. Local reconstruction applied to X-ray tomography. In IMA Volumes in Mathematics and Its Applications: Inverse Problems in Wave Propagation. Vol 90. G. Chavent, G. Papanicolaou, P. Sack, and W. Symes, editors. Springer-Verlag, New York. 443-452.

12. Chilian, W.M. 1997. Coronary microcirculation in health and disease: summary of an NHLBI workshop. Circulation. 95:522-528.

13. Kockx, M.M., G.R.Y. De Meyer, H. Bortier, N. De Meyer, J. Muhring, A. Bakker, W. Jacob, L.C. Vaeck, and A. Herman. 1996. Luminal foam cell accumulation is associated with smooth muscle cell death in the intimal thickening of human saphenous vein grafts. Circulation. 94:1255-1262.

14. Lerman, A., M.W. Webster, J.H. Chesebro, W.D. Edwards, C.M. Wei, V. Fuster, and J.C.J. Burnett. 1993. Circulating and tissue endothelin in immunoreactivity in hypercholesterolemic pigs. Circulation. 88:2923-2928.

15. Lerman, A., D.R.J. Holmes, M.R. Bell, K.N. Garratt, R.A. Nishimura and J.R.J. Burnett. 1995. Endothelin in coronary endothelial dysfunction and early atherosclerosis in humans. Circulation. 92:2426-2431.

16. Allain, C.C., L.S. Poon, C.S.L. Chan, W. Richmond, and P.C. Fu. 1974. Enzymatic determination of total serum cholesterol. Clin. Chem. 20:470-475.

17. Edelman, E.R., M.A. Nugent, L.T. Smith, and M.J. Karnovsky. 1992 Basic fibroblast growth factor enhances the coupling of intimal hyperplasia and proliferation of the vasa vasorum in injured rat arteries. J. Clin. Invest. 89:465-473.

18. Barker, S.G.E., P.A. Causton, P.A. Baskerville, S. Gent, and J.F. Martin. 1992. The vasa vasorum of the rabbit carotid artery. J. Anatomy. 180:225-231.

19. Heistad, D.D., M.L. Marcus, G.E. Larsen, and M.L. Armstrong. 1981

Role of vasa vasorum in nourishment of the aortic wall. Am. J. Physiol. 240: H781-H787.

20. Wilens, S.L., J.A. Malcon, and J.M. Vazques. 1965. Experimental infarction (medial necrosis) of the dog's aorta. Am. J. Pathol. 47:695-711.

21. Williams, J.K., and D.D. Heistad. 1996. The vasa vasorum of the arteries. J. Mal. Vasc. 21(Suppl. C):266-269.

22. Nakata, Y., and S. Shionoya. 1966. Vascular lesions due to the obstruction of vasa vasorum. Nature. 212:1258-1259.

23. Barker, S.G.E., J.E. Beesley, P.A. Baskerville, and J.F. Martin. 1995 The influence of adventitia on the presence of smooth muscle cells and macrophages in the arterial intima. Eur. J. Endovasc. Surg. 9:222-227.

24. Barker, S.G.E., L.C. Tilling, G.C. Miller, J. Beesley, G. Fleetwood, G.T. Stavri, P.A. Baskerville, and J.F. Martin. 1994. The adventitia and atherogenesis: removal initiates intimal proliferation in the rabbit which regresses on generation of "neoadventitia." Atherosclerosis. 105:131-144.

25. Geiringer, E. 1951. Intimal vascularization and atherosclerosis. $J$. Pathol. Bacteriol. 63:201-207.

26. Heistad, D.D., and M.L. Armstrong. 1986. Blood flow through vasa vasorum of coronary arteries in atherosclerotic monkeys. Arteriosclerosis. 6:326-331.

27. Werber, A., and D.D. Heistad. 1985. Diffusional support of the arteries. Am. J. Physiol. 248:H901-H909.

28. Isner, J.F. 1996. Vasa vasorum. Therapeutic implications. Cath. Cardiov. Diagn. 39:221-223.

29. Ribeiro, S.M.F., S. Schultz-Cherry, and J.E. Ullrich. 1995. Heparinbinding vitronectin up-regulates latent TGF-b production by bovine aortic endothelial cells. J. Cell. Sci. 108:1553-1561.

30. Brookes, P., R. Clark, and D. Cheresh. 1994. Requirement for vascular integrin $\mathrm{a}_{\mathrm{v}} \mathrm{b}_{3}$ for angiogenesis. Science. 264:569-571.

31. Drake, C.J., D.A. Cheresh, and C.D. Little. 1995. An antagonist of integrin $\mathrm{a}_{\mathrm{v}} \mathrm{b}_{3}$ prevents maturation of blood vessels during embryonic neovascularization. J. Cell. Sci. 108:2655-2661.

32. Waltenberger, J., U. Mayr, S. Pentz, and V. Hombach. 1996. Functional upregulation of the vascular endothelial growth factor receptor KDR by hypoxia. Circulation. 94:1647-1654.

33. Starvi, G.T., I.C. Zachary, P.A. Baskerville, J.F. Martin, and J.D. Erusalimsky. 1995. Basic fibroblast growth factor upregulates the expression of vascular endothelial growth factor in vascular smooth muscle cells. Circulation. 92: 11-14.

34. Lupu, F., D. Heim, F. Bachmann, and E.K.O. Kruithof. 1994. Expression of LDL receptor-related protein $/ \mathrm{a}_{2}$-macroglobulin receptor in human normal and atherosclerotic arteries. Arterioscler. Thromb. 14:1438-1444.

35. San-Huang, J. 1989. a a-Macroglobulins: a modulator for growth factors. Am. J. Respir. Cell Mol. Biol. 1:169-170.

36. James, K. 1990. Interactions between cytokines and $\mathrm{a}_{2}$-macroglobulin. Immunol. Today. 11:163-166.

37. Jamieson, D.G., D.C. Usher, D.J. Radier, and E. Lavi. 1995. Apolipoprotein(a) deposition in atherosclerotic plaques of cerebral vessels. A potential role for endothelial cells in lesion formation. Am. J. Pathol. 147:1567-1574.

38. Martin, J.F., R.F. Booth, and S. Moncada. 1990. Arterial wall hypoxia 
following hypoperfusion through the vasa vasorum is an initial lesion in atherosclerosis. Eur. J. Clin. Invest. 20:588-592.

39. Cuevas, P., A.M. Gonzallez, F. Carceller, and A. Baird. 1991. Vascular response to basic fibroblast growth factor when infused onto the normal adventitia or into the injured media of the rat carotid artery. Circ. Res. 69:360-369.

40. Fukuo, K., T. Inoue, S. Morimoto, T. Nakahashi, O. Yasuda, S. Kitano, R. Sasada, and T. Ogihara. 1995. Nitric oxide mediates cytotoxicity and basic fibroblast growth factor release in cultured vascular smooth muscle cells: a possible mechanism of neovascularization in atherosclerotic plaques. J. Clin. Invest.
95:669-676

41. Thomas, K.A., and G. Gimenez-Gallego. 1986. Fibroblast growth factors: broad spectrum mitogens with potent angiogenic activity. Trends Biochem. Sci. 11:81.

42. Couffinhal, T., M. Kearney, B. Witzenbichler, D. Chen, T. Murohara, W. Losordo, J. Symes, and J. Isner. 1997. Vascular endothelial growth factor/ vascular permeability factor (VEGF/VPF) in normal and atherosclerotic human arteries. Am. J. Pathol. 150:1673-1685. 